\title{
Time resolution of thin Low Gain Avalanche Detectors.
}

Riccardo Del Burgo*

Physik-Institut der Universität Zürich

E-mail: riccardo.del.burgo@cern.ch

The CMS MIP Timing Detector, proposed for the HL-LHC upgrade, will be instrumented with $\mathrm{O}(10)$ square meters of ultra-fast Silicon detectors (UFSD) in the forward region. These UFSDs are aimed at measuring the time of passage of each track with a precision of about $30 \mathrm{ps}$. The sensor that will be used for this task is the low gain avalanche detectors (LGAD). In this contribution, we will present the latest results from laboratory measurements on 50 and $35 \mu \mathrm{m}$ thick LGADs fabricated by CNM, in the framework of the AIDA-2020 project. We will concentrate on the timing performance of the sensors. Additionally the electrical characterisation of the sensor will be discussed.

7th Annual Conference on Large Hadron Collider Physics - LHCP2019

20-25 May, 2019

Puebla, Mexico

* Speaker.

${ }^{\dagger}$ F. Canelli, S. Hidalgo, B. Kilminster, A. Macchiolo, G. Pellegrini, I. Villa Alvarez. 


\section{Low Gain Avalanche Detectors (LGADs)}

LGADs are silicon sensors that exploit charge multiplication, through an internal gain layer, to enhance significantly the capability to measure track arrival times, with the potential of dramatically improving the event reconstruction process in high-energy physics experiments. The state of the art devices are $50 \mu \mathrm{m}$ or thinner. The sketch in Fig. (1) shows a cross-section of a typical segmented LGAD device.

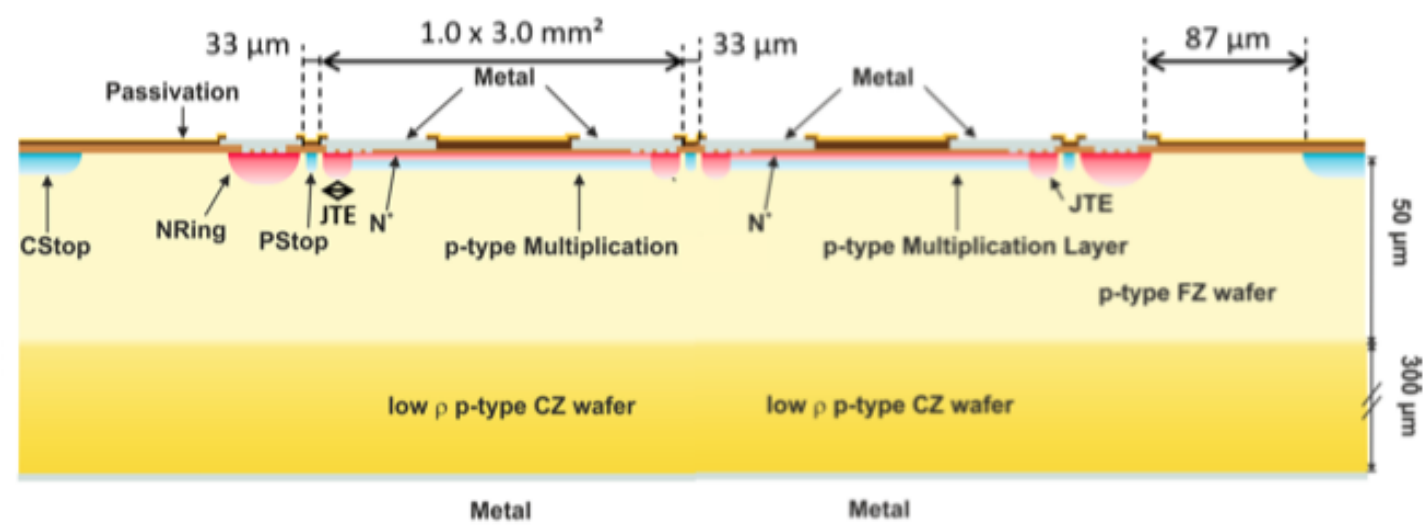

Figure 1: Sketch of a typical LGAD structure. The figure shows the different elements, as the multiplication layer, normally doped with Boron, the JTE and the p-stop to isolate the neighbouring pads.

The cross section shows the insertion in the PiN diode structure of a gain layer to produce a low controlled multiplication. The sensors capacitance is small, $\mathscr{O}(p f)$, and so the signal is fast with a rise-time in the order of hundreds of picoseconds. In order to obtain an uniform time response, the active region of the sensor is typically much larger than the thickness. On the contrary, the inter-pad regions typically represent dead areas, due to the implementation of JTE and of the p-stop structures.

The sensors investigated in this study are from a common from a common LGAD run, produced at CNM within the framework of AIDA-2020 [1].

These sensors are produced from 4" wafers and they implement different designs of the JTE termination resulting also in different inter-gap widths.

The sensors used are single pad and come in two different thickness, $35 \mu \mathrm{m}$ and $50 \mu \mathrm{m}$, and different Boron doping for the gain layer. All the sensors have a production problem and as a result they show a higher leakage current than normal. Even if the leakage current level is very high, it has been measured to be uniform among the different structures and the breakdown voltage is found to be in excess of 200V. The leakage current as a function of the bias voltage is shown in Fig. (2). As reported in section 3, the high leakage current does not have an effect on the timing performance of the sensors.

\section{Laboratory setup and time resolution measurement tecnique}

The setup used to test the time resolution is shown in Fig. (3). Two sensors, from the same 


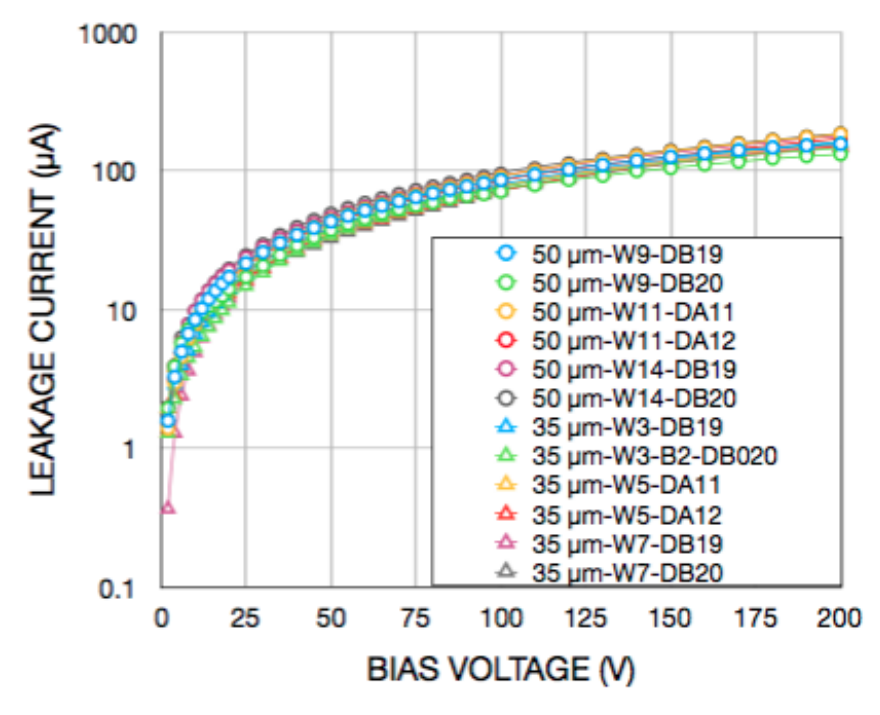

Figure 2: Leakage current of single pad devices in the AIDA-2020 run produced at CNM, as measured in a probe-station.
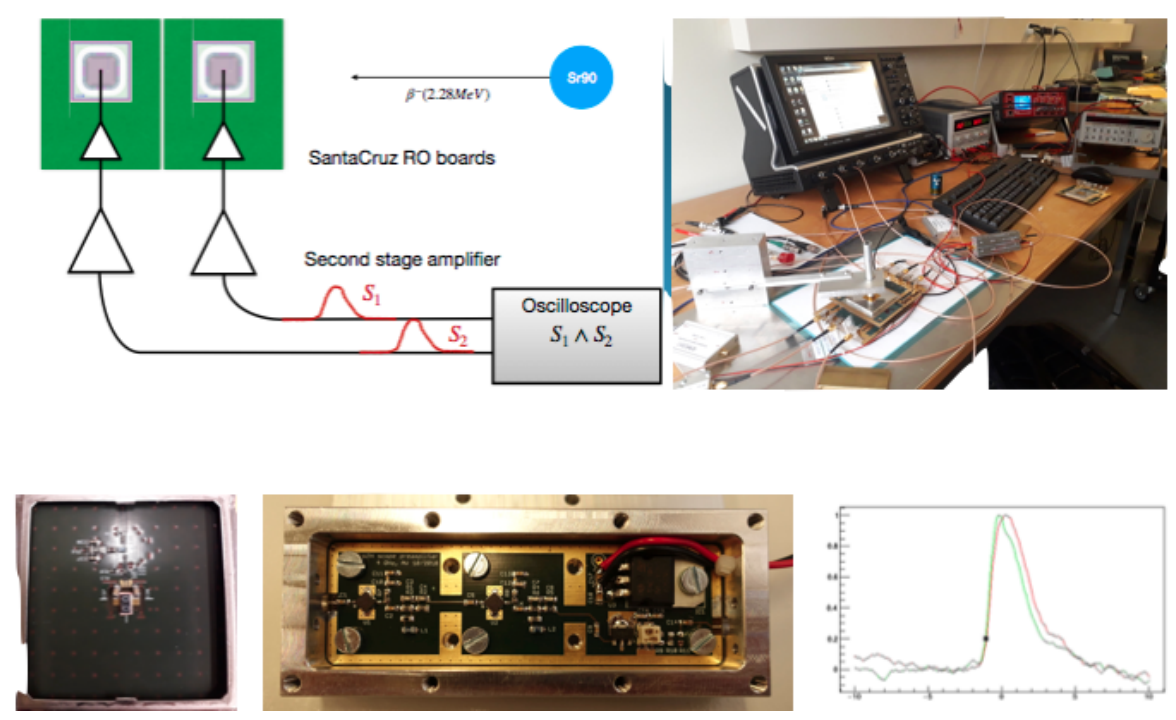

Figure 3: Setup of the timing measurements, starting from the top left figure, clockwise: schematic diagram of the measurement procedure, picture of the setup in the laboratory at $\mathrm{UZH}$, example of the signals coming from an event, the second stage amplifier and the sensor on the UCSC readout board.

wafer are wire bonded to the UCSC read-out board with a first stage amplifier [2]. The readout board is based on a single transistor common emitter design and acts as an inverting transimpedance amplifier. At a bandwidth of $1.9 \mathrm{GHz}$ a gain of $29 \mathrm{~dB}$ is expected. The sensors are then aligned one on top of the other, under a $\mathrm{Sr}^{90}$ source. The signal is then amplified with a $36 \mathrm{~dB}$ low noise high dynamic range three stage amplifier, and finally read by a fast oscilloscope ( $40 \mathrm{MS} / \mathrm{s}, 4$ GHz bandwidth). 
The beta particle the source pass through both sensors, and the time of arrival is computed for each sensor by using the CFD (Constant Fraction Discriminator) technique. The principle of this method is shown in Fig.(4) along with the constant threshold technique and two contributions to the final time resolution that will be discussed shortly below. The CFD takes into account that signals of different amplitude will cross a constant threshold at different time. To avoid this effect known as time walk, the CFD defines the time of arrival as the time at which the waveform cross a fixed fraction of the peak value of the signal. From the practical point of view each waveform is normalised using its amplitude, and the time of arrival is chosen when the waveform cross a fixed fraction $0<\mathrm{p}<1.0$. A typical value for $\mathrm{p}$ is 0.2 .

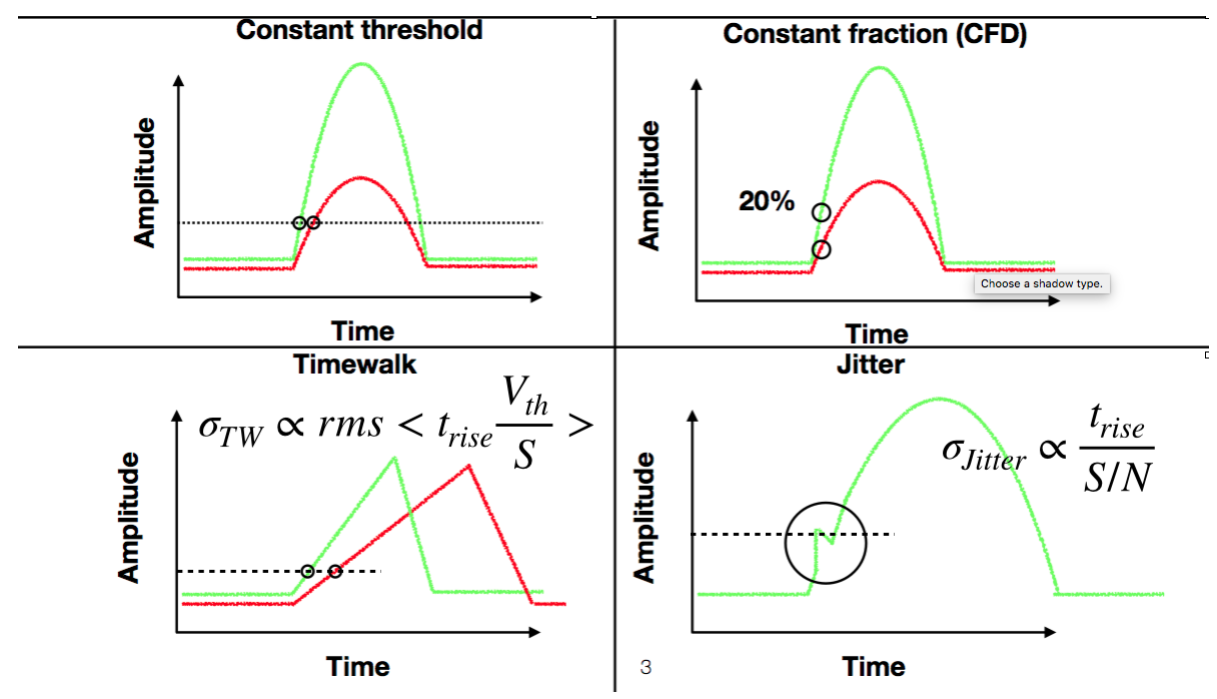

Figure 4: (top) Schematics of the Constant Threshold Discriminator method, of the Constant Fraction Discriminator method, (bottom) schematics of the Jitter and time walk effect.

Being the sensors identical, the time of arrival difference between the two sensors, for the same particle, is a measure of the time resolution of the system. The total time resolution of the system is parametrised as:

$$
\sigma=\sigma_{\text {Landau }} \bigoplus \sigma_{\text {timewalk }} \bigoplus \sigma_{\text {jitter }} \bigoplus \sigma_{\text {digitizer }}
$$

, where:

- $\sigma_{\text {Landau }}$ is the intrinsic contribution from the sensor. When a particle is passing by, the probability to create an hole-electron pair as function of time or depth follows a Landau distribution. This term is in the order of 20-30 ps, and is the biggest contribution in LGAD sensors.

- $\sigma_{\text {timewalk }}$ has been already discussed. The final contribution of this term is in the order of $\mathscr{O}(1)$ ps and is a function of the constant fraction value.

- $\sigma_{j i t t e r}$ is related to the SNR ( Signal to Noise Ratio). Noise introduces an uncertainty on when the signal is crossing the threshold. This term is the second more important in our setup, and is in the order of $\mathscr{O}(10)$ ps. 
- $\sigma_{\text {digitizer }}$ Is negligible in our setup where the sampling rate of the oscilloscope is $25 \mathrm{ps}$, and this term is calculated as $(25 / \sqrt{12})$ ps $\approx 7 \mathrm{ps}$

The final time resolution will take into account all this different contributions, some can be mitigated like the time-walk using the CFD, others like the jitter can be reduced using low-noise read-out electronic, others are intrinsic to the sensor like the Landau terms, and are irreducible.

Using the formula discussed above, the time resolution is extracted as the width of the time difference distribution divided by a factor $\sqrt{2}$.

$$
\sigma_{\text {sensor }}=\sigma_{T} / \sqrt{2}
$$

Time difference distribution

( $35 \mu \mathrm{m}$ medium doping @ 50\%,140V)

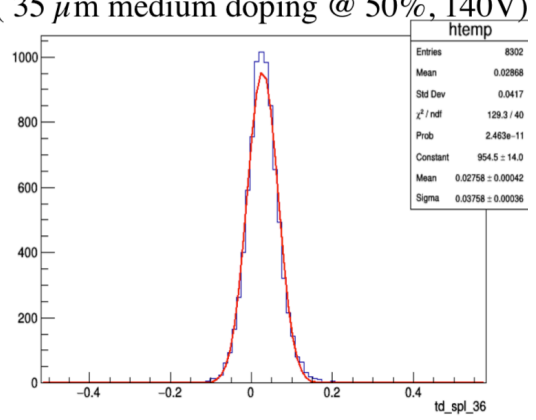

time resolution vs CFD

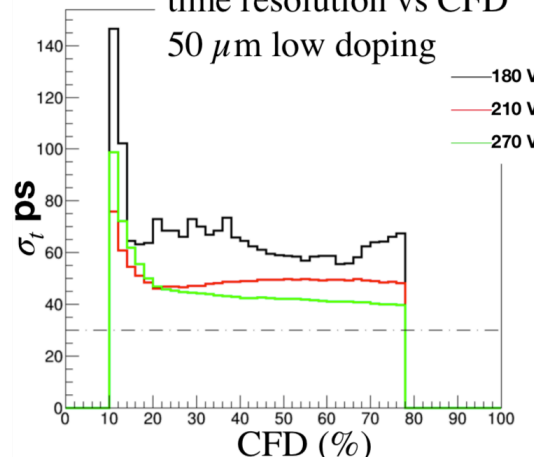

time resolution vs CFD

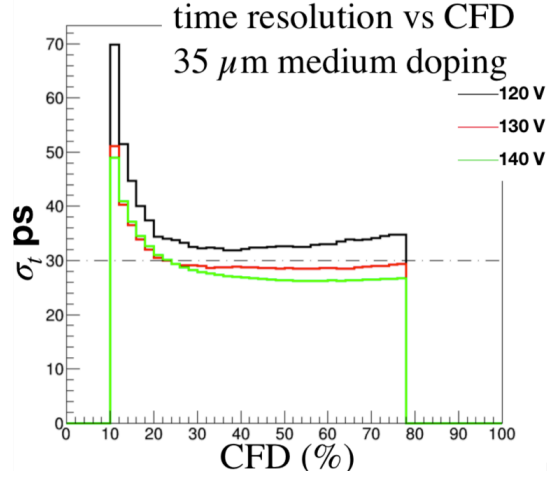

time resolution vs CFD

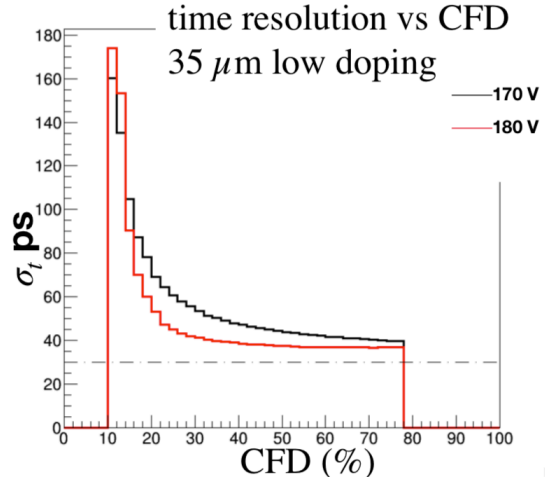

Figure 5: Top left : Example of the time difference distribution for the $35 \mu \mathrm{m}$ thick sensor with a medium doping dose. The resolution has been extracted using the CFD with a threshold of 50\% Top right: time resolution for the $35 \mu \mathrm{m}$ thick sensor, medium doping dose, as a function of the CFD threshold for different bias voltages, $120 \mathrm{~V}$ in black, $130 \mathrm{~V}$ in red and $140 \mathrm{~V}$ in green. Bottom left: time resolution for the $50 \mu \mathrm{m}$ thick sensor, low doping dose, as a function of the CFD threshold for different bias voltages, 180 in black, $210 \mathrm{~V}$ in red and $270 \mathrm{~V}$ in green. Bottom right: time resolution for the $35 \mu \mathrm{m}$ thick sensor, low doping dose, as a function of the CFD threshold for different bias voltages, $170 \mathrm{~V}$ in black, and $180 \mathrm{~V}$ in green.

\section{Results of the time resolution measurements}

The time resolution as function of the constant fraction cut has been measured for sensors with two different values of the Boron implant dose. In the case of the lower implant dose, the 
performance of $35 \mu \mathrm{m}$ and $50 \mu \mathrm{m}$ thickness has been compared.The results are summarised in Fig.(5) All the time resolution plots have a set of common features. When the constant fraction $p$ is low the SNR is quite large and the time resolution is therefore degraded. For values of $p \approx 20$ $\%$ the time resolution reaches a minimum, and for higher value of $p$ the time-walk effect begins to degrade the performances of the system. The best time resolution is obtained for the medium doping $35 \mu \mathrm{m}$ thick sensor, which achieves a $25 \mathrm{ps}$ time resolution at a bias voltage of $140 \mathrm{~V}$. The low doping sensors reach a very similar value for the time resolution, under $40 \mathrm{ps}$, but with the important difference that the bias voltage in the $35 \mu \mathrm{m}$ thick sensors is lower than the one used in the $50 \mu \mathrm{m}$ sensor ( $180 \mathrm{~V}$ vs $270 \mathrm{~V}$ ), which will be important for future practical applications.

\section{Acknowledgement}

This project has received funding from the European Union's Horizon 2020 Research and Innovation programme under Grant Agreement no. 654168.

\section{References}

[1] http://aida2020.web.cern.ch.

[2] https://twiki.cern.ch/twiki/bin/view/Main/UcscSingleChannel. 